\title{
Efficient Interval Partitioning for Constrained Global Optimization
}

Chandra Sekhar Pedamallu ${ }^{1,2}$, Linet Özdamar ${ }^{3}$, Tibor Csendes ${ }^{4}$, and Tamás Vinkó $^{5}$

1 Nanyang Technological University, School of Mechanical and Aerospace Engineering, Singapore,

2 New England Biolabs Inc., 240 County Road, Ipswich, MA pcs.murali@gmail.com

3 Yeditepe University, Dept. of Systems Engineering, Istanbul, Turkey linetozdamar@lycos.com

4 University of Szeged, Institute of Informatics, Szeged, Hungary csendes@inf.u-szeged.hu

5 ESA/ESTEC, Advanced Concepts Team, Noordwijk, The Netherlands Tamas.Vinko@esa.int.

Summary. A new efficient interval partitioning approach to solve constrained global optimization problems is proposed. This involves a new parallel subdivision direction selection method as well as an adaptive tree search. The latter explores nodes (intervals in variable domains) using a restricted hybrid depth-first and bestfirst branching strategy. This hybrid approach is also used for activating local search to identify feasible stationary points. The new tree search management technique results in improved performance across standard solution and computational indicators when compared to previously proposed techniques. On the other hand, the new parallel subdivision direction selection rule detects infeasible and suboptimal boxes earlier than existing rules, and this contributes to performance by enabling earlier reliable deletion of such subintervals from the search space.

Key words: constrained global optimization, interval partitioning, adaptive search tree management, subdivision direction selection rules, parsing.

\section{Introduction}

Many important real world problems can be expressed in terms of a set of nonlinear constraints that restrict the real domain over which a given performance criterion is optimized, that is, as a Constrained Optimization Problem (COP). A COP is defined by an objective function, $f\left(x_{1}, \ldots, x_{n}\right)$ to be maximized over a set of variables, $V=x_{1}, \ldots, x_{n}$, within respective intervals: 
$X_{i}=\left[\underline{X}_{i}, \bar{X}_{i}\right]$ for $x_{i}, i=1, \ldots, n$, that are restricted by a set of constraints represented as

$$
\begin{array}{cc}
g_{i}\left(x_{1}, \ldots, x_{n}\right) \leq 0 & i=1, \ldots, k, \\
h_{i}\left(x_{1}, \ldots, x_{n}\right)=0 & i=k+1, \ldots, r .
\end{array}
$$

These constraint can be linear and nonlinear equations or inequalities. An optimal solution of a COP is an element $x^{*}$ of the search space $X=X_{1} \times$ $\left.\cdots \times X_{n}\right)$, that meets all the constraints, and whose objective function value, $f\left(x^{*}\right) \in f(x)$ for all other consistent elements $x \in X$.

It is hard to tackle the general non-convex COP, and in general, traditional numeric algorithms cannot guarantee global optimality and completeness in the sense that the solution found might be only a local optimum. Here, we introduce an Interval Partitioning Algorithm (IP) that subdivides the continuous domain over which the COP is defined and conducts reliable assessment of the subdomains (boxes) while searching for the globally optimal solution. The reliability is meant in the sense that a box that has a potential to contain a global optimizer point is never discarded. By principle, an interval partitioning method continues to subdivide a given irrelevant box until either it turns out to be infeasible or suboptimal. Then, the box is discarded by the feasibility or optimality cutoff tests. Otherwise it becomes a small enclosure (by nested partitioning) with that possibly contains a local stationary point. During the partitioning process, an increasing number of solution candidates are identified by invoking a local search procedure in promising boxes. Hence, similar to other interval and non-interval B\&B techniques, a local search procedure is utilized in IP to find $x^{*}$ in a given box. Here, we use Feasible Sequential Quadratic Programming, FSQP, as a local search method that has convergence guarantee when started from a location nearby a stationary point.

Theoretically, IP has no difficulties in dealing with the COP; however, interval research on the $\mathrm{COP}$ is relatively scarce when compared with bound constrained optimization. An early reference is that of Robinson who used interval arithmetic to obtain bounds for the solution of the COP [Rob73]. Hansen and Sengupta first used IP to solve the inequality COP [HS80]. A detailed discussion on interval techniques for the general COP with both inequality and equality constraints is provided in Ratschek and Rokne [RR88] and Hansen [Han92], and some numerical results using these techniques have been published later in [Wol94]. Dallwig et al. proposed the software GLOPT for solving bound constrained optimization and the COP and a new reduction technique was suggested [DNS97]. Kearfott presented GlobSol, which is an IP software that is capable of solving bound constrained optimization problems and the COP [Kea03]. Markót [Mar03] developed an IP for solving the COP with inequalities where new adaptive multi-section rules and a new box selection criteria were presented [MFCC06].

Our contribution described in Section 2 to the generic IP lies in two features: a new adaptive tree search method that can be used both in noninterval and interval B\&B approaches, and a new subdivision direction selec- 
tion (branching) rule that can be used in interval methods. This new branching rule aims at reducing the uncertainty degree in the feasibility regarding the constraints over a given subdomain as well as the uncertainty in the box's potential of containing a global optimizer point. The new techniques are based on symbolic manipulation of the expressions [CR93, RC93, POC06, POC07]. While in [CR93, RC93] nonlinear transformation was applied to simplify the objective function, in [POC06, POC07] the parsing of the computation tree was introduced to have a good decision on the subdivision directions.

Our numerical experiments (in Section 4) show that the resulting IP is a viable method for these problems. The results are compared with commercial software such as BARON, MINOS, and other solvers interfaced with GAMS.

\section{Interval Partitioning Algorithm for the COP}

The set of compact intervals is denoted by $\mathbb{I}$. Every interval $X \in \mathbb{I}$ is also denoted by $[\underline{X}, \bar{X}]$, with the bounds of $\underline{X}=\min X$ and $\bar{X}=\max X$. For every $a \in R$, the point interval $[a, a]$ is also denoted by $a$. The width of an interval $X$ is the real number $w(X)=\bar{X}-\underline{X}$. Given two real intervals $X$ and $Y, X$ is said to be tighter than $Y$ if $w(X)<w(Y)$.

Elements of $\mathbb{I}^{n}$ are the boxes. Given $X_{1}, \ldots, X_{n} \in \mathbb{I}$, the corresponding box $X$ is the Cartesian product of intervals, $X=X_{1} \times \cdots \times X_{n}$, where $X \in \mathbb{I}^{n}$. A subset of $X, Y \subseteq X$, is a subbox of $X$. The notion of width is defined as

$$
w\left(X_{1} \times \cdots \times X_{n}\right)=\max _{1 \leq i \leq n} w\left(X_{i}\right) .
$$

Interval arithmetic operations are set theoretic extensions of the corresponding real operations [AH83]. Given $x, y \in \mathbb{I}$, and an operation $\diamond \in$ $\{+,-, \times, \div\}$, we have: $X \diamond Y=\{x \diamond y \mid x \in X, y \in Y\}$. Due to properties of monotonicity, these operations can be implemented by real computations over the bounds of intervals. The associative law and commutativity hold for these operations, but the distributive law does not hold (only a weaker law is true, subdistributivity).

Interval arithmetic is particularly appropriate to represent inclusion of real quantities. The range of a real function $f$ over an interval $X$ is denoted by $f(X)$, and it can be computed by interval extensions.

Definition 1. An inclusion function of a real function $f: D_{f} \subset R^{n} \rightarrow R$ is a function $F: \mathbb{I}^{n} \rightarrow \mathbb{I}$ such that $\forall X \in \mathbb{I}^{n}, X \in D_{f} \Rightarrow f(X)=\{f(x) \mid x \in X\} \subseteq$ $F(X)$.

This inclusion formula is the basis of what is called the fundamental theorem of interval arithmetic: interval extensions always produce inclusion functions that enclose the range of the corresponding real function. As a result, suppose that, for instance, you are looking for a zero of a real function $f$ over a domain $D$. If the evaluation of an interval extension of $f$ over $D$ does not 
contain 0 , it means that 0 was not part of the range of $f$ over $D$. In a proper computer implementation of interval extension based inclusion functions the outside rounding must be made to have guaranteed reliability.

The most common interval extension is known as natural extension. This is obtained by replacing each arithmetic operation and standard function found in the expression of a real function with an enclosing interval operation. Natural extension functions are inclusion isotone: given a real function $f$, whose natural extension is denoted by $F$, and two intervals $X$ and $Y$ such that $X \subseteq Y$, the following holds: $F(X) \subseteq F(Y)$. For real operations this property follows from their monotonicity. We denote the lower and upper bounds of the function interval range over a given box $Y$ by $\underline{F}(Y)$ and $\bar{F}(Y)$, respectively.

Here, it is assumed that for the studied COP, the natural interval extensions of $f, g$ and $h$ over $X$ are defined. Furthermore it is assumed that, $F$ (and similarly, $G$ and $H$ ) is $\alpha$-convergent over $X$, that is, there exist $c, \alpha>0$ such that $w(F(Y))-w(f(Y)) \leq c w(Y)^{\alpha}$ holds for all $Y \subseteq X$.

An interval constraint is built from an atomic interval formula (interval function) and relation symbols, whose semantics are extended to intervals as well. A constraint is defined by its expression (atomic formula and relation symbol), its variables, and their domains. We will consider that an interval constraint has interval variables (variables that take interval values), and that each associated domain is an interval.

The main feature of interval constraints is that if its solution set is empty, it has no solution over a given box $Y$ then it follows that the solution set of the COP is also empty and the box $Y$ can be reliably discarded. Suppose the objective function value of a feasible solution is known as the Current Lower Bound, CLB. Then, similar to infeasible boxes, suboptimal boxes can be discarded as follows. If the upper bound of the objective function range over a given box $Y$ is less than CLB, then $Y$ can be discarded since it cannot contain a better solution than the CLB.

Below we formally provide the conditions when a given box $Y$ can be discarded reliably, based on the ranges of interval constraints and the objective function. In a partitioning algorithm, each box $Y$ is assessed for its optimality and feasibility status by calculating the inclusions $F, G$, and $H$ over the domain of $Y$.

Definition 2. (Cut-off test based on optimality:) If $\bar{F}(Y)<C L B$, then the box $Y$ is called a suboptimal box.

Definition 3. (Cut-off test based on feasibility:) If $\underline{G}_{i}(Y)>0$, or $0 \notin H_{i}(Y)$ for any $i$, then box $Y$ is called an infeasible box.

Definition 4. If $\underline{F}(Y) \leq C L B$, and $\bar{F}(Y) \geq C L B$, then $Y$ is called an indeterminate box with regard to optimality. Such a box holds the potential of containing $x^{*}$ if it is not an infeasible box.

Definition 5. If $\left(\underline{G}_{i}(Y)<0\right.$, and $\left.\bar{G}_{i}(Y)>0\right)$, or $\left(0 \in H_{i}(Y) \neq 0\right)$ for some $i$, and other constraints are consistent over $Y$, then $Y$ is called an indeterminate 
box with regard to feasibility and it holds the potential of containing $x^{*}$ if it is not a suboptimal box.

Definition 6. The degree of uncertainty of an indeterminate box with respect to optimality is defined as: $P F(Y)=\bar{F}(Y)-C L B$.

Definition 7. The degree of uncertainty, $P G^{i}(Y)\left(P H^{i}(Y)\right)$ of an indeterminate inequality (equality) constraint with regard to feasibility is as: $P G^{i}(Y)=\overline{G_{i}}(Y)$, and $P H^{i}(Y)=\overline{H_{i}}(Y)+\left|\underline{H}_{i}(Y)\right|$.

Definition 8. The total feasibility uncertainty degree of a box, $I N F(Y)$, is the sum of uncertainty degrees of equalities and inequalities that are indeterminate over $Y$.

The new subdivision direction selection rule (Interval Inference Rule, IIR) targets an immediate reduction in $\operatorname{INF}(Y)$ and $P F(Y)$ and chooses the respective variables to bisect a given parent box. The IP described in the following section uses the feasibility and optimality cut-off tests in discarding boxes and applies the new rule IIR in partitioning boxes.

\subsection{Interval Partitioning Algorithm}

IP is an algorithm that subdivides indeterminate boxes to reduce $I N F(Y)$ and $P F(Y)$ by nested partitioning. The contraction and $\alpha$-convergence properties enable this. The reduction in the uncertainty levels of boxes finally lead to their elimination due to suboptimality or infeasibility while helping IP in ranking the remaining boxes in a better way.

A box that has no uncertainty with regard to feasibility after nested partitioning still has uncertainty with regard to optimality unless it is proven that it is suboptimal. The convergence rate of IP might be slow if we require nested partitioning to reduce a box to a point interval that is the global optimizer point. Hence, since a box with a high $P F(Y)$ is promising that it may contain a global optimizer, we use a local search procedure that can identify stationary points in such boxes.

Usually, IP continues to subdivide available indeterminate and feasible boxes until either they are all deleted or interval sizes of all variables in existing boxes are less than a given tolerance. Such boxes may contain a global optimizer point. Termination can also be forced by limiting the number of function evaluations and/or CPU time. Here, we choose to terminate IP when the number of function calls not counting those of the local search procedure reaches a given limit or when the CPU time exceeds a maximal allowed value. Algorithm 1 is a generic IP algorithm without calls to local search.

We now describe our new IP that has a flexible stage-wise tree management feature. This stage-wise search tree applies the best-first box selection rule within a restricted subtree (to economize memory usage), meanwhile it invokes local search in a set of boxes. 


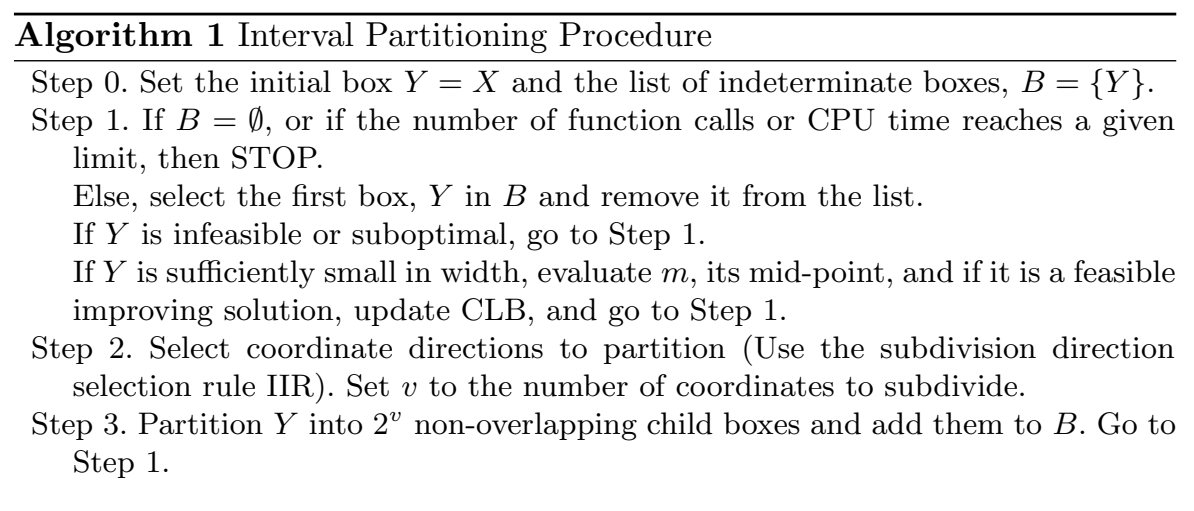

The tree management system in the proposed IP maintains a stage-wise branching scheme that is conceptually similar to the iterative deepening approach [Kor85]. The iterative deepening approach explores all nodes generated at a given search tree level (stage) before it starts assessing the nodes at the next stage. Exploration of boxes at the same stage can be done in any order, the sweep may start e.g. from best-first box of that stage. On the other hand, in the proposed adaptive tree management system, a node (parent box) at the current stage is permitted to grow a subtree forming partial succeeding tree levels and to explore nodes in this subtree before exhausting the nodes at the current stage.

If a feasible solution (and CLB) is not identified yet, boxes in the subtree are ranked according to descending $I N F(Y)$ values, otherwise they are ranked in descending order of $\bar{F}(Y)$. A box is selected among the children of the same parent according to either box selection criterion, and the child box is partitioned again continuing to build the same subtree. This subtree grows until the Total Area Deleted (TAD) by discarding boxes fails to improve in two consecutive partitioning iterations in this sub tree. Such failure triggers a call to local search where all boxes not previously subject to local search are processed by the procedure Feasible Sequential Quadratic Programming (FSQP, [ZT96]), after which they are placed back in the list of pending boxes and exploration is resumed among the nodes at the current stage. If a feasible solution with a better objective function value is found by FSQP the CLB is updated and the solution is stored.

The above adaptive tree management scheme maintains two lists of boxes, $B_{s}$ and $B_{s+1}$ that are the lists of boxes to be explored at the current stage $s$ and the next stage $s+1$, respectively. Initially, the set of indeterminate or feasible boxes in the pending list $B_{s}$ consists only of $X$ and $B_{s+1}$ is empty. As child boxes are added to a selected parent box, they are ordered according to the current criterion. Boxes in the subtree stemming from the selected parent at the current stage are explored and partitioned until there is no improvement in TAD in two consecutive partitioning iterations. 


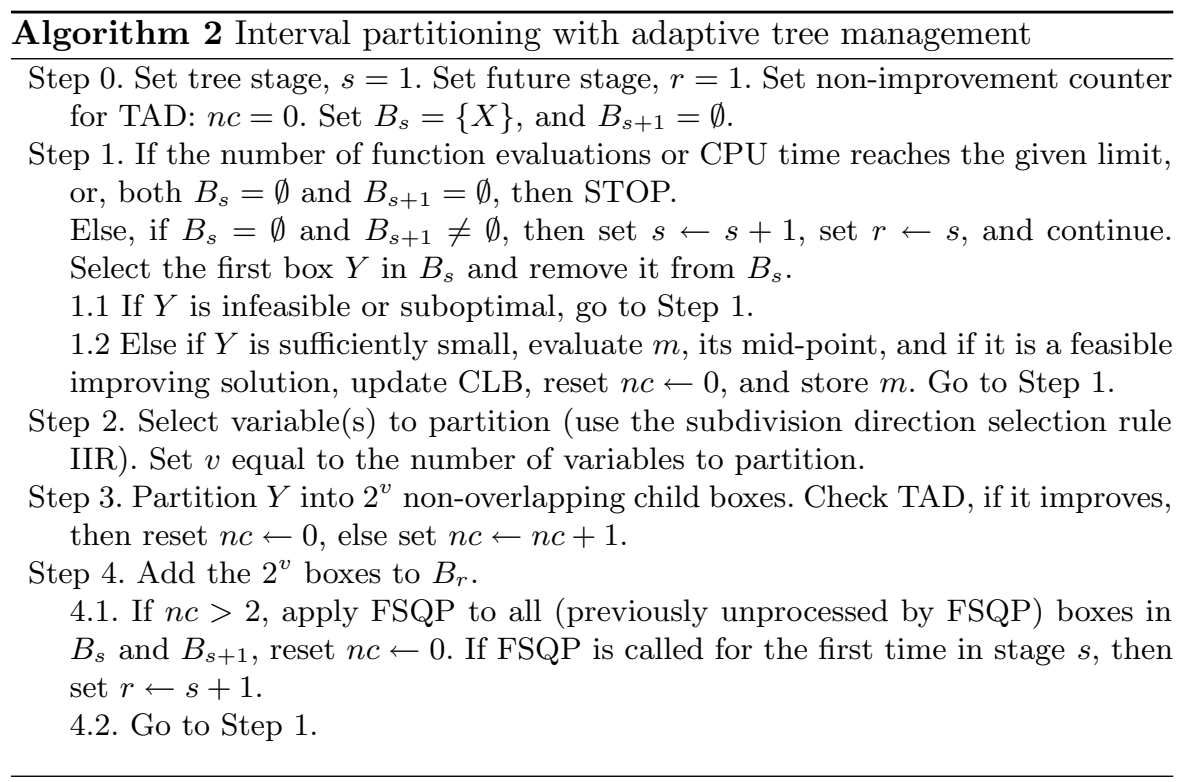

At that point, partitioning of the selected parent box is stopped and all boxes that have not been processed by local search are sent to the FSQP module and processed to identify feasible and improving point solutions if FSQP is successful in doing so. It should be noted that, whether or not FSQP fails to find an improving solution, IP will continue to partition the box since it passes both cutoff tests as long as it has a potential to contain an improving solution. Finally, the algorithm encloses potential improving solutions in sufficiently small boxes if FSQP can identify them. Thus, FSQP acts as a catalyst that occasionally scans larger boxes to identify improving solutions at the earlier stages of the search. From that moment, child boxes generated from any other selected parent in $B_{s}$ are stored in $B_{s+1}$ irrespective of further calls to FSQP in the current stage. When all boxes in $B_{s}$ have been assessed (discarded or partitioned), the search moves to the next stage, $s+1$, starting to explore the boxes stored in $B_{s+1}$.

In this manner, less boxes (those in the current stage) are maintained in memory and the search is allowed to go down to deeper levels within the same stage, increasing the chances to discard boxes. On the other hand, by enabling the search to also explore boxes horizontally across at the current stage, it might be possible to find feasible improving solutions faster by not partitioning parent boxes that are not so promising (because we are able to observe a larger number of boxes).

The tree continues to grow in this manner taking the list of boxes of the next stage after the current stage's list of boxes is exhausted. The algorithm stops either when there are no boxes remained in $B_{s}$ and $B_{s+1}$ or when there is no improvement in CLB as compared with the previous stage. The proposed 
IP algorithm is given in Algorithm 2. The adaptive tree management system in IP is illustrated in [PPC08].

\subsection{A New Subdivision Direction Selection Rule for IP}

The order in which variable domains are partitioned has an impact on the convergence rate of IP. In general, variable selection is made according to widest coordinate direction rule or largest function rate of change in the box. Here, we develop a new numerical subdivision direction selection rule, Interval Inference Rule (IIR), to improve IP's convergence rate by partitioning in parallel, those variable domains that reduce $P F(Y)$ and $I N F(Y)$ in immediate child boxes. Hence, new boxes are formed with an appropriate partitioning sequence resulting in decreased uncertainty caused by the overestimation in the indeterminate objective function range and constraint ranges.

Before IIR is applied, the objective $f$ and each constraint $g_{i}$ and $h_{j}$ are interpreted as binary trees that represent recursive subexpressions hierarchically. Such binary trees enable interval propagation over all subexpressions of the constraints and the objective function [BMV94]. Interval propagation and function trees are used by [Kea91] in improving interval Newton approach by decomposition and variable expansion, by [SP99] in automated problem reformulation, by [Sah03] and by [TS04] where feasibility based range reduction is achieved by tightening variable bounds.

After interval propagation is carried out over the subexpressions in a binary tree, IIR traverses this tree to label its nodes so as to identify the pair of variables (source variables) that are most influential on the constraint's or the objective's uncertainty degree. The presented interval subdivision direction selection rule is an alternative of earlier rules as those published in [CR97], [RC95], and [CGC00]. This pair of variables is identified for each constraint and the objective function, and placed in the pool of variables whose domains will be possibly partitioned in the next iteration. We make sure that the pool at least contains the source variables for the objective function and therefore, the number of variables to be bisected in parallel is at least two. The total pool resulting from the traversal of $f, g$ and $h$ is screened and its size is reduced by allocating weights to variables and re-assessing them.

Before the labeling process IIR-Tree can be applied on a constraint expression, it has to be parsed and intervals have to be propagated through all subexpression levels. This is achieved by calling an interval library at each subexpression level of the binary tree from bottom to top starting from atomic levels (variables or constants).

A binary tree representing a constraint is built as follows. Leaves of the binary tree are atomic elements, i.e. they are either variables or constants. All other nodes represent binary expressions of the form (Left $\Theta$ Right). A binary operator " $\Theta$ " is an arithmetic operator $(\cdot,+,-, \div)$ having two branches ("Left" and "Right") that are themselves recursive binary subtrees. However, mathematical functions such as ln, exp, sin, etc. are unary operators. For 
these the argument of the function is always placed in the "Left" branch. For a detailed example see [POC07].

When the number of constraints is large, there might be a large set of variables resulting from the application of IIR-Tree to the objective function and each constraint. Here, we develop a priority allocation scheme to narrow down the set of variables (selected by IIR-Tree) to be partitioned in parallel. In this approach, all variable pairs identified by IIR-Tree are merged into a single set $Z$. Then, a weight $w_{j}$ is assigned to each variable $x_{j} \in Z$ and the average $\bar{w}$ is calculated. The final set of variables to be partitioned is composed of the two source variables of $f$ and all other source variables $x_{j} \in Z$ with $w_{j}>\bar{w}$ belonging to the constraints.

The weight $w_{j}$ is defined as a function of several criteria: $P G^{i}(Y)$ (or $P H_{Y}^{i}$ ) of constraint $g_{i}\left(\right.$ or $h_{i}$ ), for which $x_{j}$ is identified as a source variable; the number of times $x_{j}$ exists in the expression of $g_{i}$; and the total number of multiplicative terms in which $x_{j}$ is involved within $g_{i}$. Furthermore, the existence of $x_{j}$ in a trigonometric and/or even power subexpression in $g_{i}$ is included in $w_{j}$ by inserting corresponding flag variables. When a variable $x_{j}$ is a source variable to more than one constraint, the weight calculated for each such constraint is added to result in a total weight $w_{j}$ defined as

$\sum_{i \in I C_{j}}\left(P F(Y)^{i} / P H_{\max }+P G(Y)^{i} / P G_{\max }+e_{j i} / e_{j, \max }+a_{j i} / a_{j, \max }+t_{j i}+p_{j i}\right) / 5$

where

$I C_{j}: \quad$ set of indeterminate constraints (over $Y$ ) where $x_{j}$ is a source variable,

TIC: total set of indeterminate constraints,

$P H_{\text {max }}: \max _{i \in T I C}\left\{P H^{i}(Y)\right\}$,

$P G_{\text {max }}: \max _{i \in T I C}\left\{P G^{i}(Y)\right\}$,

$e_{j i}: \quad$ number of times $x_{j}$ exists in the constraint number $i \in I C_{j}$,

$e_{j, \max }: \max _{i \in I C_{j}}\left\{e_{j i}\right\}$,

$a_{j i}$ : number of multiplicative terms $x_{j}$ is involved in constraint $i \in I C_{j}$,

$a_{j, \max }: \max _{i \in I C_{j}}\left\{a_{j i}\right\}$,

$t_{j i}$ : $\quad$ binary parameter indicating that $x_{j}$ exists in a trigonometric expression in the constraint number $i \in I C_{j}$,

$p_{j i}$ : $\quad$ binary parameter indicating that $x_{j}$ exists in an even power or abs expression in the constraint number $i \in I C_{j}$.

This weighting method is illustrated on a collection of constraints that consists of 3 constraints involving 4 variables. The three constraints are given in equations (1)-(3) below. Variable domains are $X_{1}=[-2.0,4.0], X_{2}=[0.0$, $10.0], X_{3}=[-2.0,1.0]$, and $X_{4}=[-10.0,0.0]$. 
Table 1. Inputs for calculating variable weights.

\begin{tabular}{|l|l|l|l|l|l|l|c|}
\hline Constraint No. & $x_{j}\left|e_{j i}\right| a_{j i}\left|p_{j i}\right| t_{j i}\left|\left[H_{i}(Y), \overline{H_{i}}(Y)\right]\right|$ & $P H_{Y}^{i}$ \\
\hline \hline \multirow{3}{*}{ Constraint 1 } & $x_{1}$ & 2 & 1 & 0 & 0 & & \\
& $x_{2}$ & 1 & 1 & 0 & 0 & {$[-339,261]$} & $339+261=600$ \\
& $x_{3}$ & 1 & 1 & 0 & 0 & & \\
& $x_{4}$ & 1 & 1 & 0 & 0 & & \\
\hline \multirow{3}{*}{ Constraint 2 } & $x_{1}$ & 1 & 1 & 0 & 0 & & \\
& $x_{2}$ & 1 & 1 & 0 & 0 & {$[-374,196]$} & $374+196=570$ \\
& $x_{3}$ & 2 & 1 & 0 & 0 & & \\
& $x_{4}$ & 1 & 1 & 0 & 0 & & \\
\hline \multirow{3}{*}{ Constraint 3 3} & $x_{1}$ & 3 & 3 & 1 & 1 & & \\
& $x_{2}$ & 2 & 2 & 0 & 1 & {$[-41,21]$} & $41+21=62$ \\
& $x_{3}$ & 0 & 0 & 0 & 0 & & \\
& $x_{4}$ & 1 & 1 & 0 & 0 & & \\
\hline
\end{tabular}

Table 2. A summary of the calculation of weights for source variables.

\begin{tabular}{|c|c|c|c|c|}
\hline Variable & $\begin{array}{c}\text { Weight in } \\
\text { constraint no. 1 }\end{array}$ & $\begin{array}{c}\text { Weight in } \\
\text { constraint no. 2 }\end{array}$ & $\begin{array}{c}\text { Weight in } \\
\text { constraint no. 3 }\end{array}$ & $\begin{array}{c}\text { Total variable } \\
\text { weight }\left(w_{j}\right)\end{array}$ \\
\hline \hline$x_{1}$ & 0.4 & 0.32 & 0.82 & 1.54 \\
\hline$x_{2}$ & 0.4 & 0.59 & 0.42 & 1.41 \\
\hline$x_{4}$ & 0.6 & 0.59 & 0.42 & 1.61 \\
\hline
\end{tabular}

Table 3. Total $I N F(Y)$ of child boxes resulting from partitioning different pairs of variables.

\begin{tabular}{|c|c|c|c|c|c|c|}
\hline Selected variables & $\left(x_{1}, x_{4}\right)$ & $\left(x_{1}, x_{2}\right)$ & $\left(x_{1}, x_{3}\right)$ & $\left(x_{2}, x_{3}\right)$ & $\left(x_{2}, x_{4}\right)$ & $\left(x_{3}, x_{4}\right)$ \\
\hline \hline Total $I N F(Y)$ of child boxes & $\mathbf{2 1 4 1}$ & 3088 & 2758 & 3786 & 3778 & 3728 \\
\hline
\end{tabular}

$$
\begin{aligned}
1-\left(10 x_{1}+6 x_{1} x_{2}-6 x_{3} x_{4}\right) & =0 \\
6\left(x_{1} x_{4}\right)+6\left(x_{2} x_{3}\right)-10 x_{3}-4 & =0 \\
\sin \left(x_{1} x_{2}\right) \cos \left(x_{1}^{2}-x_{2}\right)+\left(x_{1} x_{4}\right) & =0
\end{aligned}
$$

In Table 1 we provide a summary of symbolic characteristics for each variable in each constraint. Here TIC is the set of three constraints. The variable weights $w_{j}$ are calculated using the values of box- and constraint related parameters given in Table 1 . The pairs of maximum impact variables found are $\left(x_{1}, x_{2}\right),\left(x_{1}, x_{4}\right)$, and $\left(x_{1}, x_{4}\right)$ for the first, second and third constraints, respectively. The set $Z$ is $\left\{x_{1}, x_{2}, x_{4}\right\}$. A sample weight calculation for $x_{1}$ in the first constraint is given as $\left(\frac{600}{600}+\frac{2}{3}+\frac{1}{3}+\frac{0}{1}+\frac{0}{1}\right) / 5=0.4$. The weight calculations are summarized in Table 2 .

Consequently, the pair $\left(x_{1}, x_{4}\right)$ is selected for re-partitioning. This results in 3 child boxes whose total $I N F(Y)$ is indicated by bold face in the Table 3. One child box is found to be infeasible and discarded. For comparison purpose, we also show the total $I N F(Y)$ for child boxes that would result 
from re-partitioning other pairs of variables. It is observed that $\operatorname{INF}(Y)$ is the least for the pair $\left(x_{1}, x_{4}\right)$.

\section{Theoretical results}

The inclusion functions applied are obtained by natural interval extension. Hence the inclusion functions have the usual properties [AH83, RR88]: they are zero convergent $\left(\lim _{i \rightarrow \infty} w\left(X_{i}\right)=0\right.$ implies $\left.\lim _{i \rightarrow \infty} w\left(F\left(X_{i}\right)\right)=0\right)$, inclusion isotone, and $\alpha$-convergent for $\alpha=1$. The parsing is used only to determine the subdivision directions. The decrease in the inclusion function widths achieved within a single subdivision step will be characterized. Only such argument intervals are investigated that have positive width component intervals. The multiplication by the interval $[0,0]$ is excluded from the forthcoming consideration. We have proven the following four lemmas in [POC07].

Lemma 1. Let the operator $\Theta$ at any level $k$ of a binary tree be the $m$-th power ( $m$ is even) or the absolute value, and let $\Lambda^{k}=\underline{L}^{k}=0$. Further, let $\underline{L}^{k+1}<0$. Then, IIR may not able to identify $\Lambda^{k+1}$.

Lemma 2. Let trig denote any trigonometric function. Define maxtrig and mintrig as the maximum and the minimum values trig can take during one complete cycle. Further, let the operator at any level $k$ of a binary tree be $\ominus=$ trig, and maxtrig $\in\left[\underline{L}^{k}, \bar{L}^{k}\right] \bigcup\{-\infty, \infty\}$ or mintrig $\in\left[\underline{L}^{k}, \bar{L}^{k}\right] \bigcup\{-\infty, \infty\}$. Then, IIR may not be able to identify $\Lambda^{k+1}$.

Lemma 3. Suppose the interval operator at a given level $k$ is " $\odot=$ ' $\times$ ', and $\underline{L}^{k+1}, \underline{R}^{k+1}<0, \bar{L}^{k+1}, \bar{R}^{k+1}>0\left|\underline{L}^{k+1}\right|=\bar{R}^{k+1}$, and $\left|\underline{R}^{k+1}\right|=\bar{L}^{k+1}$. Then, IIR may not be able to label a bound in the right or left subtrees at the level $k+1$.

Lemma 4. For expressions excluding the ambiguous subexpressions indicated in Lemmas 1, 2, and 3, IIR identifies the correct couple of bounds at level $k+1$ that result exactly in $\Lambda^{k}$ at level $k$.

Theorem 1 below states that unless ambiguous subexpressions indicated in Lemmas 1, 2, and 3 exist in a constraint expression or in the objective function $f$, the partitioning of a parent box $Y$ along a source variable identified by IIR guarantees an immediate reduction in the labeled bound of $g_{i}, h_{i}$, or $f$. Hence, a reduction will happen in the degrees of uncertainty $\left(P G^{i}(Y), P H^{i}(Y)\right.$, or $P F(Y))$ for at least one immediate child box.

Theorem 1. Suppose that a given constraint $g_{i}(x)$ or $h_{i}(x)$, or an objective function $f$ does not contain the subexpression types indicated in Lemmas 1 , 2, and 3. Assume further that all interval components of $Y$ have a positive width and that multiplication by the interval $[0,0]$ is not allowed. Let $Y$ be a 
Table 4. Component intervals $Y_{r}^{S}$ and $Y_{m}^{S}$ for the subboxes discussed in the proof of Theorem 1.

\begin{tabular}{|c|c|c|}
\hline $\mid$ Subbox $\mid$ & $Y_{r}^{S}$ & $Y_{m}^{S}$ \\
\hline$S_{1}$ & $\left|\left[\underline{Y}_{r}, \underline{Y}_{r}+w\left(Y_{r}\right) / 2\right]\right|$ & $\left|\left[\underline{Y}_{m}, \underline{Y}_{m}+w\left(Y_{m}\right) / 2\right]\right|$ \\
\hline$S_{2}$ & $\left|\left[\underline{Y}_{r}+w\left(Y_{r}\right) / 2, \bar{Y}_{r}\right]\right|$ & $\left|\left[\underline{Y}_{m}, \underline{Y}_{m}+w\left(Y_{m}\right) / 2\right]\right|$ \\
\hline$S_{3}$ & $\left|\left[\underline{Y}_{r}, \underline{Y}_{r}+w\left(Y_{r}\right) / 2\right]\right|$ & $\left|\left[\underline{Y}_{m}+w\left(Y_{m}\right) / 2, \bar{Y}_{m}\right]\right|$ \\
\hline$S_{4}$ & $\left|\left[\underline{Y}_{r}+w\left(Y_{r}\right) / 2, \bar{Y}_{r}\right]\right|$ & $\left|\left[\underline{Y}_{m}+w\left(Y_{m}\right) / 2, \bar{Y}_{m}\right]\right|$ \\
\hline
\end{tabular}

parent box that is partitioned by at least one source variable identified by IIR. Then, $P G^{i}\left(S_{t}\right)<P G^{i}(Y), P H^{i}\left(S_{t}\right)<P H^{i}(Y)$, or $P F\left(S_{t}\right)<P F(Y)$ for at least one child $S_{t}$ of $Y$.

Proof. Consider the evaluation of $G_{i}(Y)$, the cases of the other inclusion functions can be proven similarly. We show that there is an immediate guaranteed reduction in the uncertainty degrees of three children $S_{t}, t=1, \ldots, 3$, assuming that there are two source variables, $x_{r}, x_{m}$ identified by IIR for the box $Y$ and for $g_{i}(x)$. We define $S_{1}, S_{2}, S_{3}$, and $S_{4}$ as four subboxes produced by the parallel bisection along these source variables. We denote the component intervals belonging to $x_{r}, x_{m}$ and box $Y$ as: $Y_{r}=\left[\underline{Y}_{r}, \bar{Y}_{r}\right]$ and $Y_{m}=\left[\underline{Y}_{m}, \bar{Y}_{m}\right]$, respectively. Variable domains in a given child are denoted by $Y_{j}^{S}, j=1,2, \ldots, n$. Then $Y_{j}^{S}=Y_{j}, \forall j \neq r, m$. In Table 4 , The $r$ and $m$ coordinate components of the child domains are listed.

Assume that $\bar{Y}_{r}$ and $\bar{Y}_{m}$ was identified as the source bounds most contributing to $\overline{G_{i}}(Y)$. The proof is similar for any other pair of bounds. Below we show that $P G^{i}\left(S_{t}\right)<P G^{i}(Y)$ for the children $S_{t}=S_{1}, S_{2}$, and $S_{3}$, and that $P G^{i}\left(S_{4}\right)=P G^{i}(Y)$.

Case $S_{1}$ : obviously $S_{1} \subseteq Y$. Then, by inclusion isotonicity, $w\left(G_{i}\left(S_{1}\right)\right) \leq$ $\left.w\left(G_{i}(Y)\right)\right)$ and $\left.\bar{G}_{i}\left(S_{1}\right) \leq \bar{G}_{i}(Y)\right)$. Further, since $\bar{S}_{1, r} \neq \bar{Y}_{r}$ and $\bar{S}_{1, m} \neq \bar{Y}_{m}$, then $\bar{G}_{i}\left(S_{1}\right) \neq \bar{G}_{i}(Y)$ due to the fact that $\bar{Y}_{r}$ and $\bar{Y}_{m}$ are the source bounds most contributing to $\overline{G_{i}}(Y)$. This is the point where we utilize the assumptions that all interval components have a positive width and that multiplication by the interval $[0,0]$ is not allowed.

From the above, $\bar{G}_{i}\left(S_{1}\right)<\bar{G}_{i}(Y)$ holds as strict inequality which leads to $P G^{i}\left(S_{1}\right)<P G^{i}(Y)$. One can show by similar reasoning that $P G^{i}\left(S_{2}\right)<$ $P G^{i}(Y)$ and $P G^{i}\left(S_{3}\right)<P G^{i}(Y)$. However, $P G^{i}\left(S_{4}\right)=P G^{i}(Y)$, because $\bar{S}_{4, r}=\bar{Y}_{r}$ and $\bar{S}_{4, m}=\bar{Y}_{m}$.

The above reasoning is applicable to all bound combinations of contributing source bounds not only for the $\left(\bar{S}_{r}, \bar{S}_{m}\right)$ pair. In each case, three of the children result in reduced $P G^{i}\left(S_{t}\right)$ values. When only one source variable is partitioned, the $\bar{Y}_{r}$ and $\bar{Y}_{m}$ are the most contributing source bounds to $\bar{G}_{i}(Y)$ and two children boxes are obtained, then $S_{1}$ is guaranteed to have reduced $P G^{i}$ value compared to that of $Y$. 
Based on Theorem 1, we now describe supporting rules that are applied by IIR in case labeling ambiguities described in the Lemmas are in the expressions. For subexpressions of the type indicated in Lemma 1 at level $k$ of a binary tree (with $\Lambda^{k}=\underline{L}^{k}=0$ and with such an interval bound at level $k+1$, that $\left.\underline{L}^{k+1}<0\right)$ set the bound labeling rule to be applied by IIR at level $k+1$ such that $\Lambda^{k+1}=\underline{L}^{k+1}$. That is, $\underline{L}^{k+1}$ is targeted, and hence this ambiguity will be reduced as Theorem 1 proves for other targets. As a consequence, this rule indirectly supports IIR's reduction of $I N F(Y)$ or $P F(Y)$.

Assume now that there exist a trigonometric type subexpression at level $k$ of a binary tree with maxtrig $\in\left[\underline{L}^{k}, \bar{L}^{k}\right]$ or mintrig $\in\left[\underline{L}^{k}, \bar{L}^{k}\right]$. Set the bound labeling rule to be applied by IIR at level $k+1$ such that $\Lambda^{k+1}=$ $\max \left\{\left|\underline{L}^{k+1}\right|,\left|\bar{L}^{k+1}\right|\right\}$. The ambiguity at level $k$ is resolved more and more in forthcoming partitioning iterations when $\left[\underline{L}^{k}, \bar{L}^{k}\right]$ excludes maxtrig or mintrig.

For the exceptional case handled in Lemma 3, it is the same which of the two fitting pairs of bounds is selected, the targeted value can occur only in one of its subintervals. These auxiliary rules allow us to handle also expressions involving the problematic operations characterized in Lemmas 1 to 3 . The statements of this section provide only hints that the new direction selection rules can improve the efficiency of the IP algorithm. An extensive computational testing can tell more on that.

\section{Numerical tests}

We have completed some numerical experiments with IP. First, we compare the performance of different variable selection rules (Rule A, Rule C) from the literature applicable for our IIR too. We also compare the adaptive tree management approach with two conventional tree management approaches: worst-first (we aim at discarding boxes as soon as possible) and depth-first. All three rules and the three tree management techniques are embedded in IP that uses FSQP. We also compare two box ranking approaches, the one with the swap among two criteria (maximal infeasibility $I N F(Y)$ and maximal box upper bound on the objective function $\bar{F}(Y)$ ) and a penalty approach that combines infeasibility with $f$, namely maximal upper bound of augmented $f$.

Thus, we shall measure the effectiveness of the method's different features on performance. In order to have a proper background for comparison, we considered the results of five solvers that are linked to the commercial software GAMS and a stand-alone one, FSQP [ZT96] whose code has been provided by AEM (see www. aemdesign.com/FSQPmanyobj.htm). The solvers used in this comparison are BARON 7.0 [Sah03], Conopt 3.0 [Dru96], LGO 1.0 [Pin97], MINOS 5.5 [MS87], Snopt 5.3.4 [GMS97] and FSQP. We allow every solver to complete its run without imposing additional stopping criteria except the limit on CPU time. 
Table 5. List of COP benchmarks used in the experiments: problem data and references. Dimension is denoted by $\mathrm{D}$, the number of nonlinear equations: NE, the number of linear equations: LE, the nonlinear inequalities: NIE, and the number of linear inequalities is LIE.

\begin{tabular}{|c|c|c|c|c|c|}
\hline Problem & $\begin{array}{l}\text { D, NE, LE, } \\
\text { NIE, LIE }\end{array}$ & Source & Problem & $\begin{array}{c}\text { D, NE, LE, } \\
\text { NIE, LIE }\end{array}$ & Source \\
\hline Aircraftb & $18,5,5,0,0$ & [Coco] & $\mathrm{Hs} 053$ & $5,0,3,0,0$ & {$[$ Coco $]$} \\
\hline Avgasb & $8,0,0,10,0$ & {$[\mathrm{Pri}]$} & Hs056 & $7,4,0,0,0$ & {$[\mathrm{Coco}]$} \\
\hline Alkyl & $14,6,1,0,0$ & {$[\mathrm{Coco}]$} & Hs407 & $5,3,0,0,0$ & {$[\mathrm{Coco}]$} \\
\hline Bt4 & $3,1,1,0,0$ & [Coco] & Hs108 & $9,0,0,12,0$ & {$[\mathrm{Coco}]$} \\
\hline Bt8 & $5,2,0,0,0$ & {$[\mathrm{Coco}]$} & $\mathrm{Hs} 080$ & $5,3,0,0,0$ & {$[\mathrm{Coco}]$} \\
\hline Bt12 & $5,3,0,0,0$ & [Coco] & Hs043 & $4,0,0,3,0$ & {$[\mathrm{Coco}]$} \\
\hline Bt11 & $5,2,1,0,0$ & [Coco] & Hs116 & $13,0,0,10,5$ & {$[\mathrm{Coco}]$} \\
\hline Bt7 & $5,3,0,0,0$ & [Coco] & Himmel11 & $9,3,0,0,1$ & {$[\mathrm{Coco}]$} \\
\hline Dispatch & $4,1,0,0,1$ & [Coco] & Immum & $21,0,7,0,0$ & {$[\mathrm{Coco}]$} \\
\hline Dipigri & $7,0,0,4,0$ & [Coco] & Lootsma & $3,0,0,2,0$ & {$[\mathrm{Coco}]$} \\
\hline Degenlpa & $20,0,14,0,0$ & [Coco] & Lewispol & $6,6,3,0,0$ & {$[\mathrm{Coco}]$} \\
\hline Degenlpb & $20,0,15,0,0$ & {$[\mathrm{Coco}]$} & Mwright & $5,3,0,0,0$ & {$[\mathrm{Coco}]$} \\
\hline Eig1 & $22,22,0,0,0$ & {$[\mathrm{Coco}]$} & Mhw4d & $5,3,0,0,0$ & {$[\mathrm{Coco}]$} \\
\hline Ex5 & $7,0,1,3,2$ & {$[\mathrm{Coco}]$} & Madsen & $3,0,0,6,0$ & {$[\mathrm{Coco}]$} \\
\hline Ex9 & $10,4,5,0,0$ & {$[\mathrm{Coco}]$} & Minmaxrb & $3,0,0,2,2$ & {$[\mathrm{Coco}]$} \\
\hline Ex8_4_2 & $24,10,0,0,0$ & {$[\mathrm{Coco}]$} & Median_scop_vareps & $5,0,0,3,0$ & {$[\mathrm{Coco}]$} \\
\hline Ex9_2_5 & $7,3,4,0,0,0$ & {$[\mathrm{Coco}]$} & Matrix2 & $6,0,0,2,0$ & {$[\mathrm{Coco}]$} \\
\hline $1 \_5$ & $6,0,4,2,0$ & [Coco] & Mistake & $9,0,0,12,0$ & {$[$ Coco $]$} \\
\hline Ex9_2_6 & $16,6,6,0,0$ & {$[\mathrm{Coco}]$} & O32 & $5,0,0,6,0$ & {$[\mathrm{Coco}]$} \\
\hline Ex9_2_7 & $10,4,5,0,0$ & [Coco] & Pgon & $12,0,0,15,5$ & {$[\mathrm{Coco}]$} \\
\hline Ex9_1_2 & $10,4,5,0,0$ & {$[\mathrm{Coco}]$} & Robot & $14,2,0,0,0$ & {$[\mathrm{Coco}]$} \\
\hline Ex2 & $10,0,1,0,0$ & [Coco] & $\mathrm{Rk} 23$ & $17,7,4,0,0$ & {$[\mathrm{Coco}]$} \\
\hline Ex & $13,0,0,0,9$ & {$[\mathrm{Coco}]$} & S381 & $13,0,1,0,3$ & {$[$ Pri $]$} \\
\hline Ex8_4_1 & $22,10,0,0,0$ & {$[\mathrm{Coco}]$} & S355 & $8,5,0,0,0$ & [Pri $]$ \\
\hline Ex8_4_5 & $15,11,0,0,0$ & [Coco $]$ & S336 & $3,1,1,0,0$ & [Pri] \\
\hline F_e & 4 & [Epp95] & S262 & $4,0,1,0,3$ & [Pri] \\
\hline Fern & $5,0,0,3,0$ & [Pri] & S203 & $5,3,0,0,0$ & [Pri] \\
\hline Fp_2_1 & $6,0,0,1,1$ & [Epp95] & Springs_nonconvex & $32,0,0,10,0$ & [Pri] \\
\hline & $10,0,8,0,0$ & {$[\mathrm{Coco}]$} & Steifold & $4,3,0,0,0$ & {$[\mathrm{BT} 05]$} \\
\hline & $11,4,2,0,0$ & {$[\mathrm{Co}$} & Sample & $4,0,0,2,0$ & {$[$ Pri $]$} \\
\hline Hs108 & $9,0,0,12,0$ & {$[\mathrm{Coco}]$} & $\mathrm{Hs} 080$ & $5,3,0,0,0$ & [Coco $]$ \\
\hline
\end{tabular}

\subsection{Experimental environment}

We made our extensive numerical experiments on a set of 60 COP benchmarks, five of them involving trigonometric functions. Most of these test problems are extracted from the COCONUT benchmark library [Coco] and that of the Princetonlib [Pri]. These problems are listed in the Table 5 with the number of dimensions, number of linear and nonlinear inequalities and equalities. While executing IP, we allow at most 2000 times (the number of variables + the number of constraints) function calls carried out in addition to FSQP 
calls. The number of iterations allowed for FSQP is limited to 100. We also restrict IP's run time by 900 seconds (i.e. by 2.827 Standard Time Units as defined in [Shc02]). One STU is equivalent to 318.369 seconds on our machine. All runs were executed on a PC with $256 \mathrm{MB}$ RAM, $1.7 \mathrm{GHz}$ P4 Intel CPU. The IP was coded in $\mathrm{C}++$ interfaced with the PROFIL interval arithmetic library [Knu94]. In order to illustrate the impacts of IP's individual features (the swapping double box ranking criteria, the adaptive tree search scheme and the branching rule IIR), we compared the following IP variants:

i) IP with Widest Variable Rule (Rule A, [CR96], [CR97]); IP with Rule C (also called maximum smear, [CR96], [KM90]); IP with IIR;

ii) IP with depth-first tree search approach; IP with the best-first tree search approach where box ranking is the same as that of adaptive tree approach; IP with adaptive tree management technique;

iii) IP with box ranking according to maximum $\bar{F}(Y)$ augmented with penalty, $\left(\bar{F}(Y)-I N F(Y)^{2}\right.$, described in [Yen04]); and IP with the double swapping criteria $(\max I N F(Y) / \max \bar{F}(Y))$ box ranking approach. We name the proposed double criteria approach also the no penalty approach.

The first set of IP variants listed above enable us to measure the impact of the branching rule, IIR against rules established in the interval literature. The second set of variants enable the comparison of the adaptive tree search management approach against classical tree management techniques. The third set of variants enable the comparison of the swapping double criteria box ranking method against the static penalty method. We run all three branching rules (Rule A, Rule $\mathrm{C}$, and IIR) both with the augmented $\bar{F}(Y)$ box selection approach and the proposed swapping criterion approach. All these runs used the three tree management approaches (depth-first, best-first, and adaptive). However, the depth-first approach does not require box ranking by default, hence, we have 15 different IP combinations (runs) for each test problem.

\subsection{Results}

We measured the performance of each IP variant on all benchmarks in terms of the following performance indicators: the average absolute deviation from the global optimum (abbreviated in the tables as DfO) over all 55 plus 5 benchmarks obtained within the allowed CPU time limit, the average CPU time in STUs, the average number of tree stages where IP stops (NoS), the average number of times FSQP is invoked (NoL), the average number of function calls invoked outside FSQP (NoF), the number of best solutions obtained (NoB) and the number of problems where a feasible solution could not be obtained (number of unsolved problems within the CPU time limit, NoU). We provide two summaries of results: one for the 55 non-trigonometrical problems and one for the 5 trigonometric ones. The reason for this division is that BARON is not able to solve trigonometric models. The numerical results are provided in Table 6 and 7 for non-trigonometric and trigonometric problems, respectively. 
Table 6. Summary of results obtained on 55 non-trigonometric COP benchmarks. The following abbreviations were used: average deviation from optimum: DfO, average CPU time needed in STU: STU, average number of stages: NoS, average number of FSQP calls: NoL, average number of objective function calls: NoF, number of best solutions: NoB, number of unsolved problems: NoU.

\begin{tabular}{|c|c|c|c|c|c|c|c|c|}
\hline \multicolumn{2}{|l|}{ Method } & $\mathrm{DfO}$ & STU & NoS & NoL & $\mathrm{NoF}$ & $\mathrm{NoB}$ & $\mathrm{ToU}$ \\
\hline \multirow[t]{3}{*}{ IP adaptive, no penalty } & 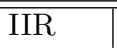 & 0.55 & 0.317 & 4.84 & 1502 & 28703 & 44 & 0 \\
\hline & Rule A & 0.61 & 0.341 & 5.82 & 1311 & 27542 & 43 & 0 \\
\hline & Rule C & 8.44 & 0.564 & 4.17 & $\begin{array}{ll}3132 \\
\end{array}$ & 28146 & 33 & 6 \\
\hline \multirow[t]{3}{*}{ IP adaptive, penalty } & IIR & 0.92 & 0.421 & 4.98 & 1382 & 25831 & 41 & 2 \\
\hline & Rule A & 2.27 & 0.458 & 5.89 & 1272 & 29824 & 39 & 1 \\
\hline & Rule C & 7.70 & 0.607 & 4.32 & 2498 & 27896 & 35 & 5 \\
\hline \multirow[t]{3}{*}{ IP best first, no penalty } & IIR & 1.83 & 1.656 & & 861 & 19402 & 39 & 3 \\
\hline & Rule A & 0.58 & 1.791 & & 894 & 22604 & 41 & 2 \\
\hline & Rule C & 3.87 & 2.467 & & 985 & 11589 & 30 & 12 \\
\hline \multirow[t]{3}{*}{ IP best first, penalty } & IIR & 0.79 & 1.847 & & 854 & 16654 & 38 & 5 \\
\hline & Rule A & 2.27 & 1.928 & & 1056 & 19678 & 40 & 1 \\
\hline & Rule C & 7.31 & 2.827 & & 982 & 11709 & 31 & 9 \\
\hline \multirow[t]{3}{*}{ IP depth first } & IIR & 0.79 & 1.847 & & 854 & 16654 & 38 & 5 \\
\hline & Rule A & 2.27 & 1.928 & & 1056 & 19678 & 40 & 1 \\
\hline & Rule C & 7.31 & 2.827 & & 982 & 11709 & 31 & 9 \\
\hline \multicolumn{2}{|l|}{$\overline{F S Q P}$} & 5.54 & 0.000 & & & & 31 & 13 \\
\hline \multicolumn{2}{|l|}{ Baron } & 0.43 & 0.067 & & & & 45 & 0 \\
\hline \multicolumn{2}{|l|}{ Conopt } & 14.54 & 0.000 & & & & 35 & 9 \\
\hline \multicolumn{2}{|l|}{ LGO } & 1.90 & 0.079 & & & & 40 & 8 \\
\hline \multicolumn{2}{|l|}{ Minos } & 14.66 & 0.000 & & & & 35 & 9 \\
\hline \multicolumn{2}{|l|}{ Snopt } & 16.36 & 0.000 & & & & 34 & 9 \\
\hline
\end{tabular}

When we compare the three tree management schemes for IP in Table 6, we observe that the overall best deviations from the optimum are obtained by IIR-adaptive tree management scheme under the no penalty box ranking scheme. This observation is also confirmed by the fact that all three rules in this configuration have the lowest number of problems where IP did not converge to a feasible solution. The performance of the widest side rule (Rule A) is close to that of IIR under adaptive tree management scheme in the no penalty box ranking approach. Rule A performs best only under best-first/no penalty box ranking configuration. In other configurations Rule $\mathrm{A}$ is inferior to IIR. Rule C (maximum smear) is usually the worst performing rule under all configurations except for the depth-first approach where it is close to IIR. In summary, best results are obtained by the IIR - adaptive - no penalty and the Rule A - best-first - no penalty combinations. However, in terms of the number of best solutions obtained, and the CPU time, IIR - adaptive - no penalty is better than its competitors. 
Table 7. Summary of results obtained on 5 trigonometric COP benchmarks. The following abbreviations were used: average deviation from optimum: DfO, average CPU time needed in STU: STU, average number of stages: NoS, average number of FSQP calls: NoL, average number of objective function calls: NoF, number of best solutions: NoB, number of unsolved problems: NoU.

\begin{tabular}{|c|c|c|c|c|c|c|c|c|}
\hline \multicolumn{2}{|l|}{ Method } & $\mathrm{DfO}$ & STU & NoS & NoL & $\mathrm{NoF}$ & $\mathrm{NoB}$ & $\mathrm{NoU}$ \\
\hline \multirow[t]{3}{*}{ IP adaptive, no penalty } & 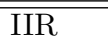 & 0.02 & 0.185 & 4.00 & 1233 & 27610 & 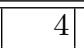 & 0 \\
\hline & Rule A & 0.03 & 0.286 & 3.60 & 1476 & 27718 & 4 & 0 \\
\hline & Rule C & 0.00 & 0.295 & 3.40 & $\begin{array}{ll}2 & 126\end{array}$ & 27684 & 4 & 1 \\
\hline \multirow[t]{3}{*}{ IP adaptive, penalty } & IIR & 0.02 & 0.251 & 5.40 & 1214 & 933 & 4 & 0 \\
\hline & Rule A & 0.03 & 0.269 & 3.80 & 1330 & 27718 & 4 & 0 \\
\hline & Rule C & 0.00 & 0.284 & 3.60 & 1818 & 27684 & 4 & 1 \\
\hline \multicolumn{2}{|c|}{ IP best first, no penalty IIR } & 0.05 & 1.739 & & 880 & 22458 & 4 & 0 \\
\hline \multirow{2}{*}{\multicolumn{2}{|c|}{$\begin{array}{l}\text { Rule A } \\
\text { Rule C }\end{array}$}} & 0.03 & 1.781 & & 800 & 14044 & 4 & 0 \\
\hline & & 0.00 & 1.311 & & 876 & 13490 & 4 & 1 \\
\hline \multirow[t]{3}{*}{ IP best first, penalty } & IIR & 0.11 & 1.711 & & 863 & 21506 & 4 & 0 \\
\hline & Rule A & 0.04 & 1.708 & & 951 & 16173 & 4 & 0 \\
\hline & Rule C & 0.00 & 2.268 & & 919 & 10337 & 4 & 1 \\
\hline \multirow[t]{3}{*}{ IP depth first } & IIR & 0.16 & 0.041 & & 387 & 27627 & 4 & 0 \\
\hline & Rule A & 0.10 & 0.032 & & 401 & 27682 & 4 & 0 \\
\hline & Rule C & 0.00 & 0.128 & & 1322 & 27718 & 4 & 1 \\
\hline \multicolumn{2}{|l|}{ FSQP } & 1.17 & 0.000 & & & & 2 & 1 \\
\hline \multicolumn{2}{|l|}{ Conopt } & 40.78 & 0.000 & & & & 1 & 0 \\
\hline \multicolumn{2}{|l|}{ LGO } & 2.23 & 0.003 & & & & 1 & 1 \\
\hline \multicolumn{2}{|l|}{ Minos } & 104.14 & 0.000 & & & & 0 & 0 \\
\hline \multicolumn{2}{|l|}{ Snopt } & 38.08 & 0.000 & & & & 1 & 0 \\
\hline
\end{tabular}

An advantage of the adaptive tree management scheme is that it reduces CPU times due to relieved memory requirements and reduced computation times due to a less number of box sorting operations as compared to the best-first approach. Furthermore, it is effective in sending the correct boxes (intervals that contain feasible solutions) to FSQP that converges to feasible solutions in a less number of iterations as compared to other tree management schemes. As expected, the best-first approach is the slowest one among all three tree management schemes (due to maintaining lengthy sorted box lists) and the fastest one is the depth-first.

However, the fastest approach is significantly inferior in solution quality in terms of unsolved problems, number of best solutions found and average absolute deviation from the optimum. For the depth-first approach, the performance of Rule C and IIR are not significantly different and that of Rule A is quite inferior. A final observation is that the no penalty box ranking method is generally better than the penalty one in both best-first and adaptive tree management approaches with respect to the sum of all three partitioning rules' average deviations from the global optimum. 
When we compare IP solvers to others, we can observe that the two complete solvers BARON and LGO are best performing. The performance of BARON is better than the best IP configuration (IIR/adaptive/no penalty) both in terms of average deviation from the optimum and CPU time. LGO's performance is somewhat inferior to that of BARON in non-trigonometric problems. The third best non-IP solver, the stand-alone FSQP, is much worse than LGO. The difference in performance between FSQP and the best IP configuration that uses FSQP as a local solver illustrates the strength of it called by a complete solver.

In the results of the trigonometric problems provided in Table 7 show that the relative performance of different IP configurations is quite similar to our findings in Table 6. The zero deviation of Rule $\mathrm{C}$ is due to its incapability of solving one problem whereas the other two rules converge in all test instances. Hence, the given average deviations of other rules are due to a single problem. Solvers other than IP are significantly inferior on these test problems as compared to any IP configuration and they do not provide the best solution in more than 2 problems out of five.

Acknowledgement. The authors wish to thank Andre Tits (University of Maryland) for providing the source code of CFSQP, and Hermann Schichl (Universität Wien) for his valuable comments and suggestions, which improved the paper.

\section{References}

[AH83] Alefeld, G. and Herzberger, J.: Introduction to Interval Computations. Academic Press, New York (1983)

[BMV94] Benhamou, F., McAllester, D. and Van Hentenryck, P.: CLP(Intervals) Revisited. Proc. of ILPS'94, pp. 124-138 (1994)

[BT05] Balogh, J., and Tóth, B.: Global optimization on Stiefel manifolds: a computational approach. CEJOR 13, 213-232 (2005)

[CGC00] Casado, L.G., García, I., Csendes, T.: A new multisection technique in interval methods for global optimization. Computing 65, 263-269 (2000)

[Coco] COCONUT, http://www.mat.univie.ac.at/ neum/glopt/coconut/

[CR93] Csendes, T., Rapcsák, T.: Nonlinear coordinate transformations for unconstrained optimization. I. Basic transformations, J. of Global Optimization 3 213-221 (1993)

[CR96] Csendes, T., Ratz, D.: A review of subdivision direction selection in interval methods for global optimization, ZAMM 76, 319-322 (1996)

[CR97] Csendes, T., Ratz, D.: Subdivision direction selection in interval methods for global optimization, SIAM J. on Numerical Analysis 34, 922-938 (1997)

[DNS97] Dallwig, S., Neumaier, A., and Schichl, H.: GLOPT - A Program for Constrained Global Optimization. In: Bomze, I.M., Csendes, T., Horst, R., Pardalos, P.M.,(eds.): Developments in Global Optimization. pp. 19-36, Kluwer, Dordrecht (1997)

[Dru96] Drud, A.S.: CONOPT: A System for Large Scale Nonlinear Optimization. Reference Manual for CONOPT Subroutine Library, ARKI Consulting and Development A/S, Bagsvaerd, Denmark (1996) 
[Epp95] Epperly, T.G.: Global optimization of nonconvex nonlinear programs using parallel branch and bound. PhD dissertation, University of Wisconsin-Madison, USA (1995)

[GMS97] Gill, P.E., Murray, W., Saunders, M.A.: SNOPT: An SQP algorithm for large-scale constrained optimization. Numerical Analysis Report 97-2, Department of Mathematics, University of California, San Diego, La Jolla, CA (1997)

[Han92] Hansen, E.R.: Global Optimization Using Interval Analysis. Marcel Dekker, New York (1992)

[HS80] Hansen, E., Sengupta, S.: Global constrained optimization using interval analysis. In: Nickel, K.L., (ed.): Interval Mathematics, Academic Press, New York (1980)

[Kea91] Kearfott, R.B.: Decompostion of arithmetic expressions to improve the behaviour of interval iteration for nonlinear systems. Computing 47, 169-191 (1991)

[Kea03] Kearfott, R.B.: An overview of the GlobSol Package for Verified Global Optimization. Talk given for the Department of Computing and Software, McMaster University, Ontario, Canada (2003)

[KM90] Kearfott, R.B., and Manuel, N.III.: A portable interval Newton/bisection package. ACM Trans. on Mathematical Software 16, 152-157 (1990)

[Knu94] Knüppel, O.: PROFIL/BIAS - A Fast Interval Library. Computing 53, 277-287 (1994)

[Kor85] Korf, R.E.: Depth-first iterative deepening: An optimal admissible tree search. Artificial Intelligence 27, 97-109 (1985)

[Mar03] Markót, M.C.: Reliable Global Optimization Methods for Constrained Problems and Their Application for Solving Circle Packing Problems. PhD dissertation, University of Szeged, Hungary (2003)

[MFCC06] Markót, M.C., Fernandez, J., Casado, L.G., Csendes, T.: New interval methods for constrained global optimization. Mathematical Programming 106, 278-318 (2006)

[MS87] Murtagh, B.A., Saunders, M.A.: MINOS 5.0 User's Guide. Report SOL 8320, Department of Operations Research, Stanford University, USA (1987)

[Ped07] Pedamallu, C.S.: New Interval Partitioning Algorithms for Global optimization problems. PhD dissertation, Nanyang Technological University, Singapore (2007)

[POC06] Pedamallu, C.S., Özdamar, L., Csendes, T.: An interval partitioning approach for continuous constrained optimization. In Models and Algorithms in Global Optimization. Springer, Berlin pp. 73-96 (2006)

[POC07] Pedamallu, C.S., Özdamar, L., Csendes, T.: Symbolic Interval Inference Approach for Subdivision Direction Selection in Interval Partitioning Algorithms. J. Global Optimization 37, 177-194 (2007)

[Pin97] Pintér, J.D.: LGO - A program system for continuous and Lipschitz global optimization. In: Bomze, I.M., Csendes, T., Horst, R., Pardalos, P.M. (eds.): Developments in Global Optimization, pp. 183-197, Kluwer, Boston (1997)

[PPC08] Pedamallu, C.S., J. Pósfai, J., Csendes, T.: Interval Partitioning Algorithm for Constraint Satisfaction Problems. Accepted for publication in the Int. J. of Modelling, Identification and Control.

[PR02] Pardalos, P.M., Romeijn, H.E.: Handbook of Global Optimization Volume 2. Nonconvex Optimization and Its Applications, Springer, Boston (2002)

[Pri] PrincetonLib.: Princeton Library of Nonlinear Programming Models. http://www. gamsworld.org/performance/princetonlib/princetonlib.htm. 
[RC93] Rapcsák, T., Csendes, T.: Nonlinear coordinate transformations for unconstrained optimization. II. Theoretical background, J. of Global Optimization 3 359-375 (1993)

[RC95] Ratz, D., Csendes, T.: On the selection of Subdivision Directions in Interval Branch-and-Bound Methods for Global Optimization. J. Global Optimization 7, 183-207 (1995)

[Rob73] Robinson, S.M.: Computable error bounds for nonlinear programming. Mathematical Programming 5, 235-242 (1973)

[RR88] Ratschek, H., Rokne, J.: New computer Methods for Global Optimization. Ellis Horwood, Chichester (1988)

[Sah03] Sahinidis, N.V.: Global Optimization and Constraint Satisfaction: The Branch-and-Reduce Approach. In: Bliek, C., Jermann, C., Neumaier, A., (eds.): COCOS 2002, LNCS 2861, 1-16 (2003)

[Shc02] Shcherbina, O., Neumaier, A., Sam-Haroud, D., Xuan-Ha Vu, Tuan-Viet Nguyen: Benchmarking global optimization and constraint satisfaction codes, LNCS 2861, 211-222 (2003)

[SP99] Smith, E.M.B., Pantelides, C.C.: A Symbolic reformulation/spatial branch and bound algorithm for the global optimization of nonconvex MINLP's. Computers and Chemical Eng. 23, 457-478 (1999)

[TS04] Tawarmalani, M., Sahinidis, N.V.: Global optimization of mixed-integer nonlinear programs: A theoretical and computational study. Mathematical Programming 99, 563-591 (2004)

[Yen04] Yeniay, O.: Penalty function methods for constrained optimization with genetic algorithms. Math. and Comp. Applications 10, 45-56 (2004)

[Wol94] Wolfe, M.A.: An Interval Algorithm for Constrained Global Optimization. J. Comput. Appl. Math. 50, 605-612 (1994)

[ZT96] Zhou, J.L., Tits, A.L.: An SQP Algorithm for Finely Discretized Continuous Minimax Problems and Other Minimax Problems with Many Objective Functions. SIAM J. on Optimization 6, 461-487 (1996) 